\title{
Article \\ Differences in Somatic Mutation Profiles between Korean Gastric Cancer and Gastric Adenoma Patients
}

\author{
Seung Woo Lee ${ }^{1}$, Taekyu Lee ${ }^{2}$, Hae Jung Sul ${ }^{3}$, Ki Cheol Park ${ }^{4}$ and Joonhong Park ${ }^{5,6, *(D)}$ \\ 1 Division of Gastroenterology, Department of Internal Medicine, College of Medicine, The Catholic University \\ of Korea, Seoul 06591, Korea; leeseungw00@hanmail.net \\ 2 Thermo Fisher Scientific Solutions, Seoul 06349, Korea; taekyu.lee@thermofisher.com \\ 3 Department of Pathology, College of Medicine, The Catholic University of Korea, Seoul 06591, Korea; \\ hjsul@cmcdj.or.kr \\ 4 Clinical Research Institute, Daejeon St. Mary's Hospital, The Catholic University of Korea, \\ Daejeon 34943, Korea; kcpark@cmcdj.or.kr \\ 5 Department of Laboratory Medicine, Jeonbuk National University Medical School and Hospital, \\ Jeonju 54907, Korea \\ 6 Research Institute of Clinical Medicine of Jeonbuk National University-Biomedical Research Institute of \\ Jeonbuk National University Hospital, Jeonju 54907, Korea \\ * Correspondence: miziro@jbnu.ac.kr; Tel.: +82-63-250-1218
}

Citation: Lee, S.W.; Lee, T.; Sul, H.J.; Park, K.C.; Park, J. Differences in Somatic Mutation Profiles between Korean Gastric Cancer and Gastric Adenoma Patients. J. Clin. Med. 2021, 10, 2038. https://doi.org/10.3390/ jcm10092038

Academic Editor: Riccardo Rossi

Received: 12 March 2021

Accepted: 7 May 2021

Published: 10 May 2021

Publisher's Note: MDPI stays neutral with regard to jurisdictional claims in published maps and institutional affiliations.

Copyright: (c) 2021 by the authors. Licensee MDPI, Basel, Switzerland. This article is an open access article distributed under the terms and conditions of the Creative Commons Attribution (CC BY) license (https:// creativecommons.org/licenses/by/ $4.0 /)$.

\begin{abstract}
Background: We aimed to investigate molecular factors potentially related to the progression of gastric adenoma (GA) to gastric cancer (GC) and compare the mutation characteristics between GC and GA. Methods: We conducted custom gene panel sequencing for 135 GC-related genes and estimated the difference in somatic mutation profiles between 20 GC and 20 GA cases. Results: A total of 31 somatic mutations, including 22 missense, 3 nonsense, and 6 frameshift mutations, were detected in 17 samples. We estimated an average of 1.8 mutations per sample (range, 1 to 3 mutations), with 12 in GC and 5 in GA. GC tended to have one or more mutated genes $(p=0.0217)$, as well as higher allele frequencies of mutated genes $(p=0.0003)$, compared to GA. Likewise, known driver mutations associated with GC tumorigenesis (TP53, ERBB2, PIK3CA, and RNF43) were identified in half of the GC cases $(50 \%, 10 / 20 ; p=0.0002)$. Only the mutant burden, regardless of gene type, was retained, with an odds ratio of 1.8392 (95\% confidence interval (CI), 1.0071 to 3.3588; $p=0.0474$ ). Conclusion: Our study demonstrates that the accumulation of mutant burden contributes to tumorigenesis progression from GA to GC in Korean patients, regardless of the kind of genes. These findings may elucidate the molecular pathogenesis of gastric carcinogenesis and malignant progression.
\end{abstract}

Keywords: mutation profiles; gastric adenoma; gastric cancer; mutant burden; next-generation sequencing

\section{Introduction}

Gastric adenoma (GA) is a precancerous lesion that is a direct precursor to gastric adenocarcinoma, a type of GC [1]. Up to 50\% of GA cases progress to gastric cancer (GC) [2], indicating the need for therapeutic removal of GAs, as well as the importance of elucidating progression mechanisms. GC shows a high mortality rate and incidence, particularly in East Asians, and approximately $90 \%$ of GCs are adenocarcinomas. Etiological and histological heterogeneity and ethnic differences complicate the genetic subtyping of GC, thus making it difficult to decide on standard treatment modalities and molecular classification systems [3]. A previous study adopting synchronous GA/GC pairs indicated that the emergence of histopathologically distinct subclones or the divergence of GA/GC pairs occurs early in gastric tumorigenesis [1]. Because of the loss of GA tissues during malignant progression, it is challenging to assess premalignant and malignant lesions in the stomach together, but residual GAs often continue to exist along with GC lesions. 
Similarly, GC in hyperplastic polyps follows a multistep progression model, a sequence from hyperplasia-dysplasia to adenocarcinoma [4]. However, it remains unknown as to whether GA includes sporadic genetic changes, and the molecular mechanisms of GA-toGC progression remain unclear. Next-generation sequencing (NGS), which allows for the interrogation of numerous variants from multiple genes within a given tumor sample, has facilitated advances in cancer evolution studies [5-7]. GA-to-GC transition is encrypted within the genome and is an evolutionary process; therefore, evolutionary perspectives on genome-wide mutational distribution and abundance may demonstrate valuable insights into GC development, with potential clinical benefits.

In this study, we aimed to investigate molecular factors potentially associated with GA-to-GC progression by comparing the mutation characteristics between GC and GA using custom gene panel sequencing.

\section{Materials and Methods}

\subsection{Study Samples}

For the sample size of this study, we referred to previous studies that investigated somatic mutations in 20 and 34 patients with GC using NGS [8,9]. Similarly to these studies, we selected 20 cases of GC and compared them with 20 cases of GA. A total of 40 formalin-fixed, paraffin-embedded (FFPE) samples of gastric tissues were obtained from 40 Korean patients diagnosed with GC (advanced $=10$, early $=10$ ) or GA classified as intestinal type only (high-grade dysplasia $(\mathrm{HGD})=10$, low-grade dysplasia $(\mathrm{LGD})=10$ ) at Daejeon St. Mary's Hospital (Daejeon, Korea). All the GC samples were obtained after surgical resection, and all the GA samples were obtained after endoscopic resection. We applied the following diagnostic criteria to classify the cancer staging and histological findings: the American Joint Committee on Cancer for cancer staging [10] and the World Health Organization (WHO) classification of tumors of the digestive system for histological findings [11]. Histological classifications from the same tissues were confirmed by a boardcertified pathologist. FFPE samples that had more than $50 \%$ tumor content to be estimated were sectioned at a thickness of 10 micrometer and preserved in $1.5 \mathrm{~mL}$ microtubes. The blade was replaced to prevent the contamination of DNA for every tissue block.

\subsection{DNA Isolation and Quantification}

Genomic DNA was isolated from four or five unstained FFPE sections per sample using the RecoverAll Total Nucleic Acid Isolation Kit (Thermo Fisher Scientific, Waltham, MA, USA) according to the manufacturer's instructions. The genomic DNA was assessed quantitatively using a Qubit 2.0 Fluorometer with a Qubit dsDNA High Sensitivity Assay Kit and a TaqMan RNase P Detection Reagent Kit (Thermo Fisher Scientific), and it was considered adequate when the DNA concentration was $>10 \mathrm{ng} / \mu \mathrm{L}$.

\subsection{Custom Panel Design and Library Preparation}

A custom panel targeting genes associated with GC or GA identified by previous research was designed using the Ion AmpliSeq Designer online tool (www.ampliseq.com, accessed on 17 April 2020). Targeted genes were chosen according to their reported associations with gastric cancer in published molecular studies (Supplementary Table S1) $[5,6,8,9,12]$. Libraries were prepared using Ion AmpliSeq Oncomine Research Panel primer pools and the Ion AmpliSeq Library Kit 2.0 Plus (Thermo Fisher Scientific) following the manufacturer's recommendation. Briefly, $20 \mathrm{ng}$ samples of genomic DNA isolates from two target amplification reactions were combined. The libraries for the custom panel were digested partially and phosphorylated using the FuPa reagent, ligated to different barcode adapters using the Ion Xpress Barcode Adapters 1-48 Kit (Thermo Fisher Scientific), and purified. The purified libraries were quantified using the Ion Library TaqMan Quantitation Kit (Thermo Fisher Scientific). 


\subsection{Sequencing Analysis Using the Ion S5XL}

Pooled purified libraries of seven multiplexed tumor DNAs per chip at a concentration of $50 \mathrm{pM}$ were loaded onto chips and analyzed using the Ion Chef with the Ion 540 chef Kit (Thermo Fisher Scientific) and sequenced on S5XL using Ion S540 chips (Thermo Fisher Scientific) for 200 base-read single-strand sequencing as per the manufacturer's instructions.

\subsection{Integrative Bioinformatic Analyses}

Automated analyses of raw sequencing data were performed sequentially in the Torrent Suite software 5.10 using default analysis parameters. Data analyses for variant calling were performed using Ion Reporter 5.10 with Oncomine Knowledgebase Reporter (https:/ /ionreporter.thermofisher.com/ir/, accessed on 23 August 2020 and the default settings. Briefly, the criterion of variant allele frequency for the preconfigured Torrent Variant Caller used the following parameters: minimum allele frequency (cutoff for supporting a variant) of InDel 0.08 and SNV 0.05; minimum coverage (total required for reads or no-call) of InDel 15 and SNV 15; and strand bias (proportion of variant alleles overwhelmingly from one strand) of InDel 0.9 and SNV 0.96. Most tumor samples were within the standards of sequencing results: mapped reads $>2,000,000$, uniformity $>85 \%$, on-target rate $>85 \%$, and mean depth $>500 \times$. Results with suspected errors and poor quality were excluded according to $<100 \times$ coverage, $<5 \%$ variant allele frequency, and variants in the out-of-coding region [13]. The final analysis of each case was reviewed and reported by a medical laboratory doctor. All genetic alterations were reported following standards and guidelines for interpreting sequence variants in cancer [14].

\subsection{Statistical Analysis}

Descriptive statistics were used to demonstrate the mean $\pm \mathrm{SD}$ of the age and mutant burden of patients. Chi-square or Fisher's exact tests were used to compare genetic differences between GC and GA according to the pathophysiological and/or histological findings. Normality was assessed using the Shapiro-Wilk test, and Student's $t$-test was used to compare the means of mutant burdens between GC and GA. Multivariate logistic regression analysis was conducted to investigate independent factors related with GA-to-GC progression. Nonsignificant predictors were removed using the enter method (probability threshold for removal: 0.1). The diagnostic performance of factors for identifying patients with GC was assessed using the area under the receiver operating characteristic (ROC) curve (AUC). Statistical analysis was performed using MedCalc Statistical Software Version 19.5.3 (MedCalc Software Ltd., Ostend, Belgium). Two-tailed $p<0.05$ was regarded to indicate statistical significance.

\section{Results}

\subsection{Clinicopathologic Difference between Gastric Cancer and Adenoma}

Upon comparing the clinicopathologic findings between GC and GA, intestinal metaplasia was found more frequently in GA than in GC $(p=0.0409)$. The clinicopathologic findings of 40 Korean patients with gastric cancer or adenoma are shown in Table 1. 
Table 1. Clinicopathologic findings of 40 Korean patients with gastric cancer or gastric adenoma.

\begin{tabular}{|c|c|c|c|c|c|c|}
\hline \multirow{2}{*}{ Findings } & \multirow{2}{*}{$\begin{array}{c}\text { Total } \\
(n=40)\end{array}$} & \multicolumn{2}{|c|}{ Gastric Cancer $(n=20)$} & \multicolumn{2}{|c|}{ Gastric Adenoma $(n=20)$} & \multirow{2}{*}{$p$ Value } \\
\hline & & 10 AGC & 10 EGC & 10 HGD & 10 LGD & \\
\hline Male & 27 & 5 & 8 & 7 & 7 & 0.7389 \\
\hline Age (Mean \pm SD), year & $70.9 \pm 10.3$ & $70.1 \pm 13.9$ & $69.7 \pm 8.1$ & $72.2 \pm 7.9$ & $71.7 \pm 11.5$ & 0.5353 \\
\hline Site & & & & & & 0.5346 \\
\hline Antrum & 20 & 3 & 6 & 6 & 5 & \\
\hline Body & 19 & 7 & 3 & 4 & 5 & \\
\hline Cardia & 1 & 0 & 1 & 0 & 0 & \\
\hline Pathologic stage & & & & & & $\mathrm{N} / \mathrm{A}$ \\
\hline I & 10 & 0 & 10 & $\mathrm{~N} / \mathrm{A}$ & $\mathrm{N} / \mathrm{A}$ & \\
\hline II & 6 & 6 & 0 & $\mathrm{~N} / \mathrm{A}$ & $\mathrm{N} / \mathrm{A}$ & \\
\hline III & 3 & 3 & 0 & $\mathrm{~N} / \mathrm{A}$ & $\mathrm{N} / \mathrm{A}$ & \\
\hline IV & 1 & 1 & 0 & $\mathrm{~N} / \mathrm{A}$ & $\mathrm{N} / \mathrm{A}$ & \\
\hline Differentiation & & & & & & $\mathrm{N} / \mathrm{A}$ \\
\hline Well & 0 & 0 & 0 & $\mathrm{~N} / \mathrm{A}$ & $\mathrm{N} / \mathrm{A}$ & \\
\hline Moderately & 11 & 4 & 7 & $\mathrm{~N} / \mathrm{A}$ & $\mathrm{N} / \mathrm{A}$ & \\
\hline Poorly & 9 & 6 & 3 & $\mathrm{~N} / \mathrm{A}$ & $\mathrm{N} / \mathrm{A}$ & \\
\hline Lauren classification & & & & & & $\mathrm{N} / \mathrm{A}$ \\
\hline Intestinal & 6 & 4 & 2 & $\mathrm{~N} / \mathrm{A}$ & $\mathrm{N} / \mathrm{A}$ & \\
\hline Diffuse & 9 & 6 & 3 & $\mathrm{~N} / \mathrm{A}$ & $\mathrm{N} / \mathrm{A}$ & \\
\hline Mixed & 5 & 0 & 5 & $\mathrm{~N} / \mathrm{A}$ & $\mathrm{N} / \mathrm{A}$ & \\
\hline Invasion site & & & & & & $\mathrm{N} / \mathrm{A}$ \\
\hline Lymphatic & 18 & 10 & 8 & $\mathrm{~N} / \mathrm{A}$ & $\mathrm{N} / \mathrm{A}$ & \\
\hline Venous & 13 & 9 & 4 & $\mathrm{~N} / \mathrm{A}$ & $\mathrm{N} / \mathrm{A}$ & \\
\hline Perineural & 6 & 5 & 1 & $\mathrm{~N} / \mathrm{A}$ & $\mathrm{N} / \mathrm{A}$ & \\
\hline Subtype of adenoma & & & & & & $\mathrm{N} / \mathrm{A}$ \\
\hline Intestinal & $\mathrm{N} / \mathrm{A}$ & $\mathrm{N} / \mathrm{A}$ & $\mathrm{N} / \mathrm{A}$ & 10 & 10 & \\
\hline Foveolar & $\mathrm{N} / \mathrm{A}$ & $\mathrm{N} / \mathrm{A}$ & $\mathrm{N} / \mathrm{A}$ & 0 & 0 & \\
\hline Gastric pyloric gland & $\mathrm{N} / \mathrm{A}$ & $\mathrm{N} / \mathrm{A}$ & $\mathrm{N} / \mathrm{A}$ & 0 & 0 & \\
\hline Intestinal metaplasia & 28 & 4 & 7 & 10 & 7 & 0.0409 \\
\hline H. pylori infection & 25 & 5 & 7 & 6 & 7 & 0.7471 \\
\hline Family history & 3 & 1 & 0 & 1 & 1 & 0.5533 \\
\hline Alcohol & 8 & 0 & 4 & 1 & 3 & 1.0000 \\
\hline Smoking & 4 & 1 & 2 & 0 & 1 & 0.2980 \\
\hline
\end{tabular}

AGC, advanced gastric cancer; EGC, early gastric cancer; HGD, gastric adenoma with high-grade dysplasia; LGD, gastric adenoma with low-grade dysplasia; N/A, not available.

\subsection{Quality Control Metrics of Raw Sequencing Data}

In quality control $(\mathrm{QC})$ metrics for raw sequencing data generated from six independent experiments, the mean number of total usable reads was $31,187,553(63 \%)$ and the mean read length was $104 \mathrm{bp}$ (SD, 8; range, 95-122). The mean mapped read count, ontarget read rate, mean depth of on-target regions, and uniformity were 5,423,026 bp, $95 \%$, $898 \times$, and $91.7 \%$, respectively. All experiments satisfied the manufacturer's specifications ( $>95 \%$ of amplicons should have a read depth of $>500 \times$ ).

\subsection{Somatic Mutation Profiles}

A total of 4178 unfiltered variants were identified from the raw sequencing data using the Ion AmpliSeq custom panel. After variant filtering of cancer genes to determine potential genes of interest, 31 somatic SNVs or indels passed the data analysis algorithms. Among the 31 somatic mutations, 22 missense, 3 nonsense, and 6 frameshift mutations were detected in the 135 GC-related genes. Details of the somatic mutation profiles in 17 Korean patients with GC or GA are summarized in Table 2. We detected an average of 1.8 mutations per sample (range, 1 to 3 mutations) in 12 GC and 5 GA cases. The five most commonly mutated genes were TP53 $(n=6)$, APC $(n=4)$, ERBB2 $(n=3)$, PIK3CA $(n=3)$, and RNF43 $(n=3)$. TP53 was the most commonly mutated gene, with mutations found in three AGC and three EGC. The APC mutation was the second most frequent, found in one 
GC and three GA. The third most commonly mutated genes were ERBB2, PIK3CA, and RNF43, which were shared in seven GC and one GA. Collectively, 11 of the 12 GC cases with any mutations had somatic mutations in TP53, ERBB2, PIK3CA, or RNF43 (Figure 1).

Table 2. Details of somatic mutation profiles in 17 Korean patients with gastric cancer or gastric adenoma.

\begin{tabular}{|c|c|c|c|c|c|c|c|c|}
\hline SN & Dx & Gene & Transcript & Base Change & AA Change & Effect & AF (\%) & COSMIC ID \\
\hline DJ01 & AGC & $M Y C$ & NM_002467.6 & c. $404 \mathrm{~A}>\mathrm{C}$ & p.Asp135Ala & Missense & 27.8 & COSM9213747 \\
\hline DJ01 & AGC & TP53 & NM_000546.5 & c. $578 \mathrm{~A}>\mathrm{G}$ & p.His193Arg & Missense & 37.7 & COSM10742 \\
\hline DJ02 & AGC & RNF43 & NM_017763.6 & c.367G > C & p.Ala123Prp & Missense & 19.3 & COSM4755837 \\
\hline DJ02 & AGC & TP53 & NM_000546.5 & c.730_740del & p.Gly244Profs*16 & Frameshift & 31.3 & $\mathrm{~N} / \mathrm{A}$ \\
\hline DJ03 & AGC & NF1 & NM_000267.3 & c. $1264 \mathrm{G}>\mathrm{A}$ & p.Ala422Thr & Missense & 13.3 & COSM3729095 \\
\hline DJ03 & AGC & $E R B B 2$ & NM_004448.3 & c. $2263 \mathrm{~T}>\mathrm{A}$ & p.Leu755Met & Missense & 29.2 & COSM1205571 \\
\hline DJ03 & AGC & PIK3CA & NM_006218.4 & c. $1035 \mathrm{~T}>\mathrm{A}$ & p.Asn345Lys & Missense & 24.8 & COSM754 \\
\hline DJ04 & AGC & FBXW7 & NM_0043303.6 & c. $1138 \mathrm{G}>\mathrm{A}$ & p.Asp380Asn & Missense & 6.5 & COSM6197887 \\
\hline DJ04 & AGC & MYC & NM_002467.6 & c. $221 \mathrm{C}>\mathrm{T}$ & p.Pro74Leu & Missense & 18.6 & COSM1166663 \\
\hline DJ07 & AGC & $A P C$ & NM_000038.6 & c. $775 \mathrm{C}>\mathrm{T}$ & p.Arg259Trp & Missense & 9.9 & COSM2990952 \\
\hline DJ07 & AGC & PIK3CA & NM_006218.4 & c. $1636 \mathrm{C}>\mathrm{T}$ & p.Gln546* & Nonsense & 23.2 & COSM24712 \\
\hline DJ09 & AGC & DDR2 & NM_006182.4 & c. $398 \mathrm{G}>\mathrm{A}$ & p.Arg133Gln & Missense & 13.3 & COSM6922479 \\
\hline DJ09 & AGC & KRAS & NM_033360.4 & c. $35 \mathrm{G}>\mathrm{T}$ & p.Gly12Val & Missense & 21.2 & COSM520 \\
\hline DJ09 & AGC & TP53 & NM_000546.5 & c. $374 \mathrm{C}>\mathrm{G}$ & p.Thr125Arg & Missense & 21.6 & COSM45243 \\
\hline DJ10 & AGC & $E R B B 2$ & NM_004448.3 & c.2315_2320dup & p.Val773_Met774insAsnVal & Frameshift & 33.4 & COSM20959 \\
\hline DJ10 & AGC & IDH1 & NM_005896.3 & c. $367 \mathrm{G}>\mathrm{A}$ & p.Gly123Arg & Missense & 27.5 & COSM96922 \\
\hline DJ11 & EGC & RNF43 & NM_017763.6 & c.1977delT & p.Ser661Profs*39 & Frameshift & 12.0 & COSM1734865 \\
\hline DJ11 & EGC & TP53 & NM_000546.5 & c. $856 \mathrm{G}>\mathrm{A}$ & p.Glu286Lys & Missense & 20.1 & COSM10726 \\
\hline DJ12 & EGC & EGFR & NM_005228.5 & c. $1474 \mathrm{~A}>\mathrm{G}$ & p.Ser492Gly & Missense & 7.9 & COSM236671 \\
\hline DJ12 & EGC & PIK3CA & NM_006218.4 & c. $3127 \mathrm{~A}>\mathrm{T}$ & p.Met1043Leu & Missense & 23.8 & COSM5731063 \\
\hline DJ13 & EGC & TP53 & NM_000546.5 & c. $916 \mathrm{C}>\mathrm{T}$ & p.Arg306* & Nonsense & 11.4 & COSM10663 \\
\hline DJ15 & EGC & ROS1 & NM_002944.2 & c. $5854 \mathrm{G}>\mathrm{T}$ & p.Gly1952* & Nonsense & 16.1 & $\mathrm{~N} / \mathrm{A}$ \\
\hline DJ16 & EGC & $E R B B 2$ & NM_004448.3 & c.2313_2314insCATTAC & p.Ala771_Tyr772insHisTyr & Frameshift & 7.6 & COSM20959 \\
\hline DJ16 & EGC & TP53 & NM_000546.5 & c. $432 \mathrm{G}>\mathrm{T}$ & p.Gln $144 \mathrm{His}$ & Missense & 19.1 & COSM45076 \\
\hline DJ22 & HGD & $A P C$ & NM_000038.6 & c. $3358 \mathrm{G}>\mathrm{A}$ & p.Gly1120Arg & Missense & 11.6 & COSM19329 \\
\hline DJ24 & HGD & KRAS & NM_033360.4 & c. $351 \mathrm{~A}>\mathrm{C}$ & p.Lys117Asn & Missense & 8.3 & COSM19940 \\
\hline DJ24 & HGD & RNF43 & NM_017763.6 & c.2228_2229del & p. Gly743Alafs*3 & Frameshift & 29.6 & COSM8520865 \\
\hline DJ28 & HGD & ROS1 & NM_002944.2 & c. $6095 \mathrm{G}>\mathrm{C}$ & p. Gly2032Ala & Missense & 5.9 & COSM6012441 \\
\hline DJ33 & LGD & $A P C$ & NM_000038.6 & c.1742delA & p.Lys581Argfs*9 & Frameshift & 7.5 & COSM1432181 \\
\hline DJ35 & LGD & $A P C$ & NM_000038.6 & c. $688 \mathrm{C}>\mathrm{T}$ & p.Arg230Cys & Missense & 7.1 & COSM1696039 \\
\hline DJ35 & LGD & FBXW7 & NM_004958.4 & c. $1647 \mathrm{~T}>\mathrm{A}$ & p.Phe549Leu & Missense & 6.9 & COSM9232927 \\
\hline
\end{tabular}

$\mathrm{SN}$, sample number; Dx, diagnosis; AA, amino acid; AF, allele frequency; COSMIC ID, Catalogue of Somatic Mutations in Cancer Internal Database; AGC, advanced gastric cancer; EGC, early gastric cancer; HGD, gastric adenoma with high-grade dysplasia; LGD, gastric adenoma with low-grade dysplasia; N/A, not available.

\subsection{Genetic Differences between Gastric Cancer and Adenoma}

Because the histopathology of GC differs from that of GA, genetic differences between GC and GA were investigated. GC had one or more mutated genes $(p=0.0217)$, as well as a higher allele frequency of mutated genes $(p=0.0003)$, compared to GA. Likewise, for GC, known driver mutations associated with GC tumorigenesis (TP53, ERBB2, PIK3CA, or RNF43) were identified in half of the GC samples $(50 \%, 10 / 20 ; p=0.0002)$ (Table 3$)$. In an enter stepwise logistic regression model, only the mutant burden, regardless of the kind of gene, was retained with an odds ratio of 1.8392 (95\% confidence interval (CI), 1.0071 to 3.3588; $p=0.0474$ ). The ROC curve analysis for allele frequency of mutated genes showed significant diagnostic utility with an AUC of 0.842 ( $95 \%$ CI, 0.718 to 0.927$)$ for GA-to-GC progression (Figure 2). 


\begin{tabular}{|c|c|c|c|c|c|c|c|c|c|c|c|c|c|c|c|c|c|}
\hline \multirow{3}{*}{ Genes } & \multicolumn{12}{|c|}{ Gastric cancer } & \multicolumn{5}{|c|}{ Gastric adenoma } \\
\hline & \multicolumn{7}{|c|}{$\operatorname{AGC}(n=7)$} & \multicolumn{5}{|c|}{$\operatorname{EGC}(n=5)$} & \multicolumn{3}{|c|}{$\operatorname{HGD}(n=3)$} & \multicolumn{2}{|c|}{$\operatorname{LGD}(n=2)$} \\
\hline & DJ01 & DJ02 & DJ03 & Dj04 & Dj07 & DJ09 & DJ10 & DJ11 & DJ12 & DJ13 & DJ15 & DJ16 & DJ22 & DJ24 & DJ28 & DJ33 & DJ35 \\
\hline \multicolumn{18}{|l|}{$A P C$} \\
\hline \multicolumn{18}{|l|}{$D D R 2$} \\
\hline \multicolumn{18}{|l|}{ EGFR } \\
\hline \multicolumn{18}{|l|}{$E R B B 2$} \\
\hline \multicolumn{18}{|l|}{ FBXW7 } \\
\hline \multicolumn{18}{|l|}{$I D H 1$} \\
\hline \multicolumn{18}{|l|}{ KRAS } \\
\hline \multicolumn{18}{|l|}{$M Y C$} \\
\hline \multicolumn{18}{|l|}{$N F 1$} \\
\hline \multicolumn{18}{|l|}{ PIK3CA } \\
\hline \multicolumn{18}{|l|}{$R N F 43$} \\
\hline \multicolumn{18}{|l|}{ ROS1 } \\
\hline$T P 53$ & & & & & & & & & & & & & & & & & \\
\hline
\end{tabular}

Figure 1. Distribution of somatic mutation profiles based on disease subtypes in 17 patients with gastric cancer or adenoma. Genes are indicated on the far left column, and each patient (DJOO) is depicted on the third row. AGC, advanced gastric cancer; EGC, early gastric cancer; HGD, gastric adenoma with high-grade dysplasia; LGD, gastric adenoma with low-grade dysplasia. Indigo, missense mutation; red, nonsense mutation; yellow, frameshift mutation.

Table 3. Somatic mutation profiles in 17 Korean patients with gastric cancer or gastric adenoma.

\begin{tabular}{|c|c|c|c|c|c|c|}
\hline \multirow{2}{*}{ Findings } & \multirow{2}{*}{$\begin{array}{c}\text { Total } \\
(n=17)\end{array}$} & \multicolumn{2}{|c|}{ Gastric Cancer $(n=20)$} & \multicolumn{2}{|c|}{ Gastric Adenoma $(n=20)$} & \multirow{2}{*}{$p$ Value } \\
\hline & & 10 AGC & 10 EGC & 10 HGD & 10 LGD & \\
\hline Mutated genes & & & & & & 0.0217 \\
\hline 0 & 23 & 3 & 5 & 7 & 8 & \\
\hline 1 & 5 & 0 & 2 & 2 & 1 & \\
\hline$\geq 2$ & 12 & 7 & 3 & 1 & 1 & \\
\hline $\begin{array}{l}\text { Mutant } \\
\text { burdens \% } \\
\text { (Mean } \pm \mathrm{SD} \text { ) }\end{array}$ & $17.9 \pm 9.2$ & $23.9 \pm 7.8$ & $14.8 \pm 6.0$ & $8.1 \pm 2.6$ & $7.2 \pm 0.3$ & 0.0003 \\
\hline Mutation type * & & & & & & 0.5294 * \\
\hline Missense & 22 & 13 & 4 & 3 & 2 & \\
\hline Nonsense & 3 & 1 & 2 & 0 & 0 & \\
\hline Frameshift & 6 & 2 & 2 & 1 & 1 & \\
\hline $\begin{array}{l}\text { Recurrent } \\
\text { mutations }\end{array}$ & & & & & & $0.0002 * *$ \\
\hline$A P C$ & 4 & 1 & 0 & 1 & 2 & \\
\hline$E R B B 2$ & 3 & 2 & 1 & 0 & 0 & \\
\hline PIKЗСA & 3 & 2 & 1 & 0 & 0 & \\
\hline RNF43 & 3 & 1 & 1 & 1 & 0 & \\
\hline ТP53 & 6 & 3 & 3 & 0 & 0 & \\
\hline
\end{tabular}

* Actual number of mutations was enumerated, but not number of patients. ${ }^{* *}$ Recurrent mutations of ERBB2, PIK3CA, RNF43, and TP53 except $A P C$ genes were compared. AGC, advanced gastric cancer; EGC, early gastric cancer; HGD, gastric adenoma with high-grade dysplasia; LGD, gastric adenoma with low-grade dysplasia; SD, standard deviation. 


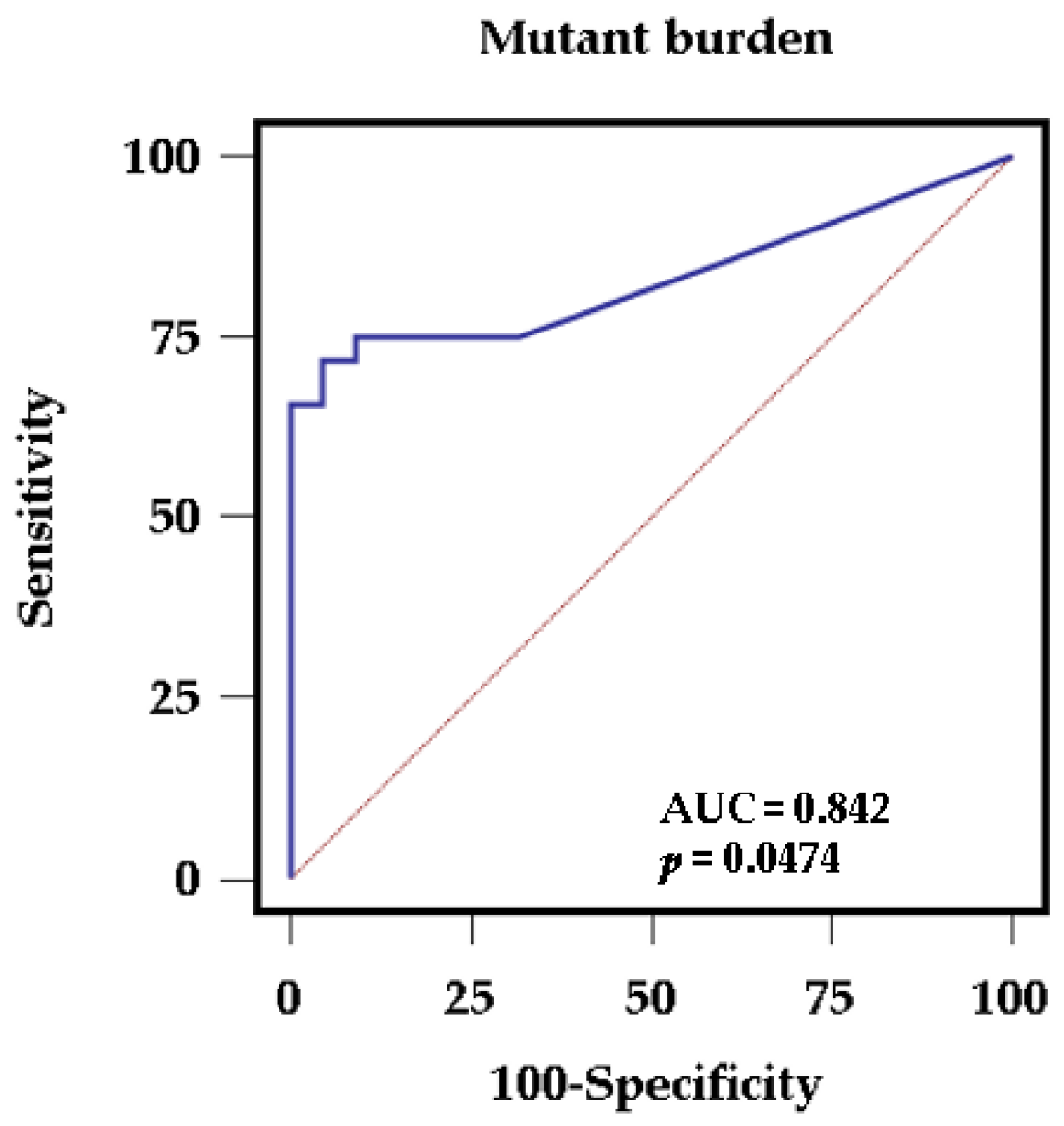

Figure 2. Receiver operating characteristic (ROC) curve for mutant burden for genetic differences between gastric cancer and gastric adenoma. The area under the curve (AUC) for mutant burden for the progression of gastric adenoma to gastric cancer is 0.842 with sensitivity of $72 \%$ and specificity of $95 \%(p=0.0474)$.

\section{Discussion}

GA is defined as localized polypoid proliferation of dysplastic epithelium of the stomach considered to represent neoplastic lesions with malignant potential, and endoscopic submucosal dissection (ESD) is the standard management for GA. The clinical signs of GA are not specific. It usually found incidentally during screening endoscopy. The WHO defines gastric adenoma as the presence of histologic unequivocal neoplastic epithelium without evidence of tissue invasion [11]. The subtypes of GA, based on their epithelial phenotypes, are intestinal type, gastric pyloric gland, and foveolar type adenoma. However, only GA classified as intestinal type was included in our study. Gastric dysplasia is divided into LGD and HGD on the basis of the degree of architectural distortion, cytoplasmic differentiation, mitotic activity, and nuclear atypia. The rate of malignant change ranges up to $23 \%$ for LGD and from 60 to $85 \%$ for HGD. The risks of metachronous HGD and metachronous gastric neoplasm (MGN) or GC after ESD did not differ between patients with LGD and those with HGD, despite the high risk reported in the HGD group. Otherwise, the HGD group showed substantially increased risk of GC or metachronous HGD compared to the LGD group in patients with no $\mathrm{H}$. pylori infection [15]. The progression intervals are 4 to 48 months for HGD and 10 to 48 months for LGD [13]. GA with an absence of polarity of proliferating cells may favor carcinoma development, and tumorassociated macrophage number may be an independent risk factor, suggesting carcinoma development regardless of the follow-up duration [16]. Key molecular events may occur during early malignant transformation and may be recorded in somatic mutation profiles from GA-to-GC progression. The current management of dysplasia is ESD according to the recent guidelines [17-19]. The life expectancy of GC depends on the stage at the time of 
diagnosis. The 5-year survival rate of early GC is excellent at more than $90 \%$; on the other hand, that of advanced GC with metastatic disease is less than 30\% [20]. First-line standard treatment includes platinum compounds and fluoropyrimidines with trastuzumab for patients with HER2-positive GC [21]. Several alternative therapies for recurrent GC are available, such as ramucirumab, a monoclonal therapeutic antibody that inhibits VEGFmediated tumor angiogenesis by binding with VEGFR2, alone or in combination with other cancer drugs [22]. However, 30 to $80 \%$ of the patients respond to treatment with ramucirumab or its combinations [23], suggesting that pharmacotherapy personalization is required to improve the efficacy of drug treatment.

In this study, we performed cancer gene panel sequencing to estimate differences in somatic mutation profiles in Korean patients with GC and GA. As a result, the number of mutated genes and mutant burden were increased in GC compared to GA. Consistent with the previous studies [1,24,25], the well-established somatic mutations TP53, APC, ERBB2, PIK3CA, RNF43, FBWX7, KRAS, MYC, and ROS1 were recurrently detected in GAs or GCs in our study, suggesting that these mutations may contribute to potential drivers of early gastric tumorigenesis. Comparing the mutations detected in our nonsynchronous GAs with GCs with those in nonsynchronous GAs [26,27], half of the GC cases had exclusively somatic mutations associated with GC tumorigenesis identified in genes such as TP53, ERBB2, PIK3CA, or RNF43. However, only mutant burden, regardless of the kind of gene, was retained by logistic regression analysis. Similarly to our study, previous research did not identify any somatic changes in hyperplastic polyp components, even in genome-wide analyses comparing hyperplastic polyps and GA, in contrast to the adenocarcinoma components [7]. In a previous study on synchronous GA/GC pairs [1], most GA/GC pairs demonstrated parallel evolution with early divergence rather than stepwise evolution during GA-to-GC progression. Nonclonal synchronous GA/GC is frequent and the obtained GA genomes already have obvious genomic changes, suggesting that attention should be paid in the diagnosis of synchronous GA and GC, particularly in recurrent or residual cases. Genetic heterogeneity affects key tumorigenesis in cancer evolution, driving phenotypic variation, and a major cause of genetic heterogeneity in cancer is genomic instability. Genetic and epigenetic alterations, as well as altered tumor microenvironments, result in tumors made up of diverse subclones with different genetic and phenotypic characteristics. Diverse subclones can establish their cooperation through paracrine, cell-cell contact, and microenvironment remodeling, which allows them to exhibit a fitness advantage during tumor progression [28,29].

In a previous molecular analysis of GC [30], TP53-inactive and TP53-active GC included patients with intermediate recurrence rates and prognosis compared to the other two subtypes, and the TP53-active GC exhibited better prognosis. The oncogenic function of mutp53 is mainly caused by alterations in the structure and properties of mutp53 that allow binding with other oncogenic or tumor suppressive proteins [31]. Interestingly, five out of six GC cases with oncogenic TP53 mutations such as p.Thr125Arg, p.Gln144His, p.His193Arg, p.Gly244Profs*16, and p.Glu286Lys in the DNA-binding domains had other somatic mutations of different genes, including ERBB2, KRAS, MYC, and RNF43. Consistent with frequent TP53 mutation, elevated expression of TP53 and aneuploidy were presented in the chromosomal instability (CIN) subtype [25]. Mouse models demonstrate that the genetic reconstitution of tumor suppression functions in the wild-type p53 rescues tumor growth. As promising therapeutic strategies, inhibitors of signaling pathways were modulated aberrantly by oncogenic mutant p53 proteins [32]. Somatic PIK3CA mutations have been reported in up to $25 \%$ of sporadic GCs and common molecular alterations in the Wnt and PI3K/Akt signaling pathways. The ratio of patients with high MSI was substantially lower, and EBV-positive and high-MSI characteristics seemed mutually inclusive in patients with GC $[3,25]$. We also found that there were three PIK3CA mutations in GC only. Inconsistent with the results of our study, gastric hyperplastic polyps with pyloric-type dysplasia were related to a PIK3CA mutation, whereas foveolar dysplasia carried TP53 mutations [33]. Gene amplifications of CCND1, CDK12, CCNE1, and ERBB2 
were identified in patients with low tumor mutational burden [3]. We identified three $E R B B 2$ mutations representing missense or frameshift mutations that were identified in GC only. The amplification status of $E R B B 2$ and other genes should be investigated due to intratumoral heterogeneity of ERBB2 amplification as a critical factor [34]. Particularly, the systemic therapeutic options for advanced GC have evolved rapidly to incorporate targeted molecular therapy with biomarker selection. In advanced GC overexpressing HER2, a combination of trastuzumab with platinum-based chemotherapy has become a standard treatment as a front-line therapy [35]. In addition, three RNF43 mutations were identified in different histopathologic samples from AGC, EGC, and GA with HGD. RNF43 mutation plays a key role as a tumorigenic driver from adenoma to carcinoma in early gastric carcinogenesis, and mutation in the tumor suppressor RNF43 and dysregulated Wnt signaling are involved in multistep gastric carcinogenesis through the adenomato-carcinoma sequence [27]. Cells lacking the functional RNF43 protein do not react to either radiation treatment or chemotherapy, and they may also accumulate additional mutations that could further aggravate disease prognosis and outcomes. Thus, RNF43 could serve as a significant GC biomarker to increase the ability to expect responses to adjuvant chemotherapy and thereby improve prognostic outcomes within the context of personalized medicine [36].

Contrary to colorectal adenocarcinoma, gastric adenocarcinoma predominantly arises from adenoma precursors and harbors truncating $A P C$ mutations, while only a minority of differentiated GCs contain $A P C$ mutations. This suggests that most gastric adenocarcinomas do not arise from adenoma precursors [37]. In our study, even though APC mutations were detected more frequently in GA $(n=3)$ than in GC $(n=1)$, the difference was not significant. APC mutations were mutually exclusive, which is consistent with their ability to activate Wnt- $\beta$-catenin signaling [38]. Similar to a previous study [27], we found that no GA or GC cases possessed both APC and RNF43 mutations. The function of the APC gene in chromosomal stability and mitosis might disappear, and G1 might be arrested, with a high quantity of DNA in the S phase. Particularly, APC mutations alter cell cycle regulation and protein expression in the diffuse type of gastric adenocarcinoma [39].

Several limitations characterize this study. First, our samples were not matched pairs of GA and GC that originated from the same individual. Thus, intertumoral bias may have affected our ability to precisely investigate spatiotemporal genetic diversity. Contrary to our expectations, the numbers of putative mutations and copy number alterations were not significantly different between GC and GA. Recently, in paired continuous lesions representing gastric tumorigenesis, cancer-like changes occurred in low-grade intraepithelial neoplasia and accumulated in high-grade intraepithelial neoplasia and early GC during intestinal-type GC tumorigenesis. The signatures of five genes, TIMP1, PLEKHS1, RGN, $L A M P 3$, and GADD45B, detected from the tumorigenesis process demonstrated robust prognostic significance for survival and relapse in GC patients, and this might result in the generation of potential molecular targets for the development of precision therapy [40]. Furthermore, transcriptome analysis using NGS may reveal potential molecular mechanisms underlying ramucirumab resistance and allow us to personalize the prescription of ramucirumab for GC [23]. Second, we did not assess MSI status associated with TP53 or the presence of EBV infection related to PIK3CA. Future studies should investigate whether differences between GC and GA are affected by the occurrence of MSI or EBV infection. Third, the numbers of cases of each histopathological subtype were relatively small, and the patients were drawn from a single center, although each GC and GA subtype was well classified. Our results may therefore not be representative of the whole population of Korean GC and GA patients. Moreover, it is possible that no calls of mutations in the remaining 23 studied patients were due to a low tumor burden carrying "actual" mutations, even though FFPE samples had more than $50 \%$ tumor content to be estimated. Further molecular analysis is required to discover the mutational contribution to gastric tumorigenesis involving larger cohorts of samples with carefully curated clinically based data coupled with detailed histological data and complemented by functional analysis. 


\section{Conclusions}

In conclusion, our study demonstrates that the accumulation of mutant burden contributes to the progression of tumorigenesis from GA to GC in Korean patients, regardless of the kind of genes. These findings may elucidate the molecular pathogenesis of gastric carcinogenesis and malignant progression. The discovery of diverse molecular characteristics by comprehensive NGS demonstrates more possibilities for both immunotherapies and targeted therapies of patients with GC arising from GA.

Supplementary Materials: The following are available online at https://www.mdpi.com/article/ $10.3390 / \mathrm{jcm} 10092038 / \mathrm{s} 1$, Table S1: A list of 135 selected genes associated with gastric cancer or adenoma for next-generation panel sequencing.

Author Contributions: Conceptualization, S.W.L.; methodology, T.L. and K.C.P.; software, T.L.; validation, J.P.; formal analysis, H.J.S. and J.P.; investigation, J.P.; data curation, S.W.L.; writingoriginal draft preparation, J.P.; writing - review and editing, S.W.L. and J.P.; supervision, S.W.L. and J.P.; project administration, S.W.L. and J.P.; funding acquisition, S.W.L. and J.P. All authors have read and agreed to the published version of the manuscript.

Funding: This work was supported by the National Research Foundation of Korea (NRF) grant funded by the Korean government (MSIT) (2020R1F1A1077316) and was supported by a clinical research institute grant funded by The Catholic University of Korea Daejeon St. Mary's Hospital (CMCDJ-P-2019-017).

Institutional Review Board Statement: This study protocol was approved by the Institutional Review Board of the Catholic University of Korea (Code number: DC19SESI0092; Date of approval: 22 October 2019).

Informed Consent Statement: Informed consent was obtained from all subjects involved in the study.

Data Availability Statement: The data presented in this study are available on request from the corresponding author.

Conflicts of Interest: The authors declare no conflict of interest.

\section{References}

1. Jung, S.-H.; Kim, S.Y.; An, C.-H.; Lee, S.-H.; Jung, E.S.; Park, H.-C.; Kim, M.S.; Chung, Y.-J.; Lee, S.H.; Kim, S.Y. Clonal Structures of Regionally Synchronous Gastric Adenomas and Carcinomas. Clin. Cancer Res. 2018, 24, 4715-4725. [CrossRef] [PubMed]

2. Abraham, S.C.; Park, S.J.; Lee, J.-H.; Mugartegui, L.; Wu, T.-T. Genetic alterations in gastric adenomas of intestinal and foveolar phenotypes. Mod. Pathol. 2003, 16, 786-795. [CrossRef] [PubMed]

3. Yu, P.; Wang, Y.; Yu, Y.; Wang, A.; Huang, L.; Zhang, Y.; Liu, W.; Wu, H.; Yao, M.; Du, Y.A.; et al. Deep Targeted Sequencing and Its Potential Implication for Cancer Therapy in Chinese Patients with Gastric Adenocarcinoma. Oncologist 2021, 26, 756-768. [CrossRef] [PubMed]

4. Imura, J.; Hayashi, S.; Ichikawa, K.; Miwa, S.; Nakajima, T.; Nomoto, K.; Tsuneyama, K.; Nogami, T.; Saitoh, H.; Fujimori, T. Malignant transformation of hyperplastic gastric polyps: An immunohistochemical and pathological study of the changes of neoplastic phenotype. Oncol. Lett. 2014, 7, 1459-1463. [CrossRef]

5. Cho, S.Y.; Park, J.W.; Liu, Y.; Park, Y.S.; Kim, J.H.; Yang, H.; Um, H.; Ko, W.R.; Lee, B.I.; Kwon, S.Y.; et al. Sporadic Early-Onset Diffuse Gastric Cancers Have High Frequency of Somatic CDH1 Alterations, but Low Frequency of Somatic RHOA Mutations Compared With Late-Onset Cancers. Gastroenterology 2017, 153, 536-549.e26. [CrossRef]

6. Cai, H.; Jing, C.; Chang, X.; Ding, D.; Han, T.; Yang, J.; Lu, Z.; Hu, X.; Liu, Z.; Wang, J.; et al. Mutational landscape of gastric cancer and clinical application of genomic profiling based on target next-generation sequencing. J. Transl. Med. 2019, 17, 189. [CrossRef]

7. Takayama, Y.; Ono, Y.; Mizukami, Y.; Itoh, H.; Nakajima, N.; Arai, H.; Tanaka, S.; Nobusawa, S.; Yokoo, H.; Onozato, Y. Comparative genome-wide analysis of gastric adenocarcinomas with hyperplastic polyp components. Virchows Archiv für Pathologische Anatomie Physiologie für Klinische Medizin 2019, 475, 383-389. [CrossRef]

8. Bria, E.; Pilotto, S.; Simbolo, M.; Fassan, M.; De Manzoni, G.; Carbognin, L.; Sperduti, I.; Brunelli, M.; Cataldo, I.; Tomezzoli, A.; et al. Comprehensive molecular portrait using next generation sequencing of resected intestinal-type gastric cancer patients dichotomized according to prognosis. Sci. Rep. 2016, 6, 22982. [CrossRef]

9. Hirotsu, Y.; Kojima, Y.; Okimoto, K.; Amemiya, K.; Mochizuki, H.; Omata, M. Comparison between two amplicon-based sequencing panels of different scales in the detection of somatic mutations associated with gastric cancer. BMC Genom. 2016, 17, 833. [CrossRef] 
10. Edge, S.B.; Compton, C.C. The American Joint Committee on Cancer: The 7th Edition of the AJCC Cancer Staging Manual and the Future of TNM. Ann. Surg. Oncol. 2010, 17, 1471-1474. [CrossRef]

11. Nagtegaal, I.D.; Odze, R.D.; Klimstra, D.; Paradis, V.; Rugge, M.; Schirmacher, P.; Washington, M.K.; Carneiro, F.; Cree, I.A.; the WHO Classification of Tumours Editorial Board. The 2019 WHO classification of tumours of the digestive system. Histopathology 2020, 76, 182-188. [CrossRef]

12. Ge, S.; Li, B.; Li, Y.; Li, Z.; Liu, Z.; Chen, Z.; Wu, J.; Gao, J.; Shen, L. Genomic alterations in advanced gastric cancer endo-scopic biopsy samples using targeted next-generation sequencing. Am. J. Cancer Res. 2017, 7, 1540-1553.

13. Kwon, D.; Kim, B.; Shin, H.C.; Kim, E.J.; Ha, S.Y.; Jang, K.-T.; Kim, S.T.; Lee, J.; Kang, W.K.; Park, J.O.; et al. Cancer Panel Assay for Precision Oncology Clinic: Results from a 1-Year Study. Transl. Oncol. 2019, 12, 1488-1495. [CrossRef]

14. Li, M.M.; Datto, M.; Duncavage, E.J.; Kulkarni, S.; Lindeman, N.I.; Roy, S.; Tsimberidou, A.M.; Vnencak-Jones, C.L.; Wolff, D.J.; Younes, A.; et al. Standards and Guidelines for the Interpretation and Reporting of Sequence Variants in Cancer. J. Mol. Diagn. 2017, 19, 4-23. [CrossRef]

15. Kim, Y.-I.; Park, J.Y.; Kim, B.J.; Hwang, H.W.; Hong, S.A.; Kim, J.G. Risk of metachronous gastric neoplasm occurrence during intermediate-term follow-up period after endoscopic submucosal dissection for gastric dysplasia. Sci. Rep. 2020, 10, 1-9. [CrossRef]

16. Taniyama, D.; Taniyama, K.; Kuraoka, K.; Zaitsu, J.; Saito, A.; Nakatsuka, H.; Sakamoto, N.; Sentani, K.; Oue, N.; Yasui, W. Long-term follow-up study of gastric adenoma; tumor-associated macrophages are associated to carcinoma development in gastric adenoma. Gastric Cancer 2017, 20, 929-939. [CrossRef]

17. Goddard, A.F.; Badreldin, R.; Pritchard, D.M.; Walker, M.M.; Warren, B.; British Society of Gastroenterology. The management of gastric polyps. Gut 2010, 59, 1270-1276. [CrossRef]

18. Dinis-Ribeiro, M.; Areia, M.; De Vries, A.C.; Marcos-Pinto, R.; Monteiro-Soares, M.; O'Connor, A.; Pereira, C.; Pimentel-Nunes, P.; Correia, R.; Ensari, A.; et al. Management of precancerous conditions and lesions in the stomach (MAPS): Guideline from the European Society of Gastrointestinal Endoscopy (ESGE), European Helicobacter Study Group (EHSG), European Society of Pathology (ESP), and the Sociedade Portuguesa de Endoscopia Digestiva (SPED). Endoscopy 2011, 44, 74-94. [CrossRef]

19. Evans, J.A.; Chandrasekhara, V.; Chathadi, K.V.; Decker, G.A.; Early, D.S.; Fisher, D.A.; Foley, K.; Hwang, J.H.; Jue, T.L.; Lightdale, J.R.; et al. The role of endoscopy in the management of premalignant and malignant conditions of the stomach. Gastrointest. Endosc. 2015, 82, 1-8. [CrossRef]

20. Jeon, J.; Cheong, J.-H. Clinical Implementation of Precision Medicine in Gastric Cancer. J. Gastric Cancer 2019, 19, $235-253$. [CrossRef]

21. Bang, Y.-J.; Van Cutsem, E.; Feyereislova, A.; Chung, H.C.; Shen, L.; Sawaki, A.; Lordick, F.; Ohtsu, A.; Omuro, Y.; Satoh, T.; et al. Trastuzumab in combination with chemotherapy versus chemotherapy alone for treatment of HER2-positive advanced gastric or gastro-oesophageal junction cancer (ToGA): A phase 3, open-label, randomised controlled trial. Lancet 2010, 376, 687-697. [CrossRef]

22. Fuchs, C.S.; Shitara, K.; Di Bartolomeo, M.; Lonardi, S.; Al-Batran, S.-E.; Van Cutsem, E.; Ilson, D.H.; Alsina, M.; Chau, I.; Lacy, J.; et al. Ramucirumab with cisplatin and fluoropyrimidine as first-line therapy in patients with metastatic gastric or junctional adenocarcinoma (RAINFALL): A double-blind, randomised, placebo-controlled, phase 3 trial. Lancet Oncol. 2019, 20, 420-435. [CrossRef] [PubMed]

23. Sorokin, M.; Poddubskaya, E.; Baranova, M.; Glusker, A.; Kogoniya, L.; Markarova, E.; Allina, D.; Suntsova, M.; Tkachev, V.; Garazha, A.; et al. RNA sequencing profiles and diagnostic signatures linked with response to ramucirumab in gastric cancer. Mol. Case Stud. 2020, 6. [CrossRef] [PubMed]

24. Wang, K.; Yuen, S.T.; Xu, J.; Lee, S.P.; Yan, H.H.N.; Shi, S.T.; Siu, H.C.; Deng, S.; Chu, K.M.; Law, S.; et al. Whole-genome sequencing and comprehensive molecular profiling identify new driver mutations in gastric cancer. Nat. Genet. 2014, 46, 573-582. [CrossRef] [PubMed]

25. The Cancer Genome Atlas Research Network Comprehensive molecular characterization of gastric adenocarcinoma. Nat. Cell Biol. 2014, 513, 202-209. [CrossRef]

26. Lim, C.-H.; Cho, Y.K.; Kim, S.W.; Choi, M.-G.; Rhee, J.-K.; Chung, Y.-J.; Lee, S.-H.; Kim, T.-M. The chronological sequence of somatic mutations in early gastric carcinogenesis inferred from multiregion sequencing of gastric adenomas. Oncotarget 2016, 7 , 39758-39767. [CrossRef] [PubMed]

27. Min, B.-H.; Hwang, J.; Kim, N.K.D.; Park, G.; Kang, S.Y.; Ahn, S.; Ahn, S.; Ha, S.Y.; Lee, Y.K.; Kushima, R.; et al. Dysregulated Wnt signalling and recurrent mutations of the tumour suppressorRNF43in early gastric carcinogenesis. J. Pathol. 2016, $240,304-314$. [CrossRef] [PubMed]

28. Burrell, R.A.; McGranahan, N.; Bartek, J.; Swanton, C. The causes and consequences of genetic heterogeneity in cancer evolution. Nat. Cell Biol. 2013, 501, 338-345. [CrossRef]

29. Zhou, H.; Neelakantan, D.; Ford, H.L. Clonal cooperativity in heterogenous cancers. Semin. Cell Dev. Biol. 2017, 64, 79-89. [CrossRef]

30. Cristescu, R.; Lee, J.; Nebozhyn, M.; Kim, K.-M.; Ting, J.C.; Wong, S.S.; Liu, J.; Yue, Y.G.; Wang, J.; Yu, K.; et al. Molecular analysis of gastric cancer identifies subtypes associated with distinct clinical outcomes. Nat. Med. 2015, 21, 449-456. [CrossRef]

31. Kim, M.P.; Lozano, G. Mutant p53 partners in crime. Cell Death Differ. 2018, 25, 161-168. [CrossRef] 
32. Blandino, G.; Di Agostino, S. New therapeutic strategies to treat human cancers expressing mutant p53 proteins. J. Exp. Clin. Cancer Res. 2018, 37, 1-13. [CrossRef]

33. Salomao, M.; Luna, A.M.; Sepulveda, J.L.; Sepulveda, A.R. Mutational analysis by next generation sequencing of gastric type dysplasia occurring in hyperplastic polyps of the stomach. Exp. Mol. Pathol. 2015, 99, 468-473. [CrossRef]

34. Tajiri, R.; Ooi, A.; Fujimura, T.; Dobashi, Y.; Oyama, T.; Nakamura, R.; Ikeda, H. Intratumoral heterogeneous amplification of ERBB2 and subclonal genetic diversity in gastric cancers revealed by multiple ligation-dependent probe amplification and fluorescence in situ hybridization. Hum. Pathol. 2014, 45, 725-734. [CrossRef]

35. Selim, J.H.; Shaheen, S.; Sheu, W.-C.; Hsueh, C.-T. Targeted and novel therapy in advanced gastric cancer. Exp. Hematol. Oncol. 2019, 8, 1-23. [CrossRef]

36. Noto, J.M.; Peek, R.M. RNF43: A Biomarker with Potential Ramifications for Therapeutic Intervention in Gastric Cancer. Cell. Mol. Gastroenterol. Hepatol. 2021, 11, 1202-1203. [CrossRef]

37. Lee, J.-H.; Abraham, S.C.; Kim, H.-S.; Nam, J.-H.; Choi, C.; Lee, M.-C.; Park, C.-S.; Juhng, S.-W.; Rashid, A.; Hamilton, S.R.; et al. Inverse Relationship between APC Gene Mutation in Gastric Adenomas and Development of Adenocarcinoma. Am. J. Pathol. 2002, 161, 611-618. [CrossRef]

38. Madan, B.; Virshup, D.M. Targeting Wnts at the Source-New Mechanisms, New Biomarkers, New Drugs. Mol. Cancer Ther. 2015, 14, 1087-1094. [CrossRef]

39. Ghatak, S.; Chakraborty, P.; Sarkar, S.R.; Chowdhury, B.; Bhaumik, A.; Kumar, N.S. Novel APC gene mutations associated with protein alteration in diffuse type gastric cancer. BMC Med Genet. 2017, 18, 61. [CrossRef]

40. Zhang, Y.; Wu, X.; Zhang, C.; Wang, J.; Fei, G.; Di, X.; Lu, X.; Feng, L.; Cheng, S.; Yang, A. Dissecting expression profiles of gastric precancerous lesions and early gastric cancer to explore crucial molecules in intestinal-type gastric cancer tumorigenesis. J. Pathol. 2020, 251, 135-146. [CrossRef] 